\title{
Macular oedema in Kawasaki syndrome
}

\author{
Justus G. Garweg • Claudia Suter • Christine Aebi-Ochsner • Reto Villiger
}

Received: 20 September 2011 /Revised: 11 December 2011 / Accepted: 19 December 2011 /Published online: 6 January 2012

(C) Springer-Verlag 2012

\section{Introduction}

Kawasaki syndrome (KS) is a rare disorder, which most frequently affects infants younger than 5 years of age. The disease is a unique form of acute systemic vasculitis, which affects primarily medium-sized, and particularly the coronary arteries. Although the pathophysiology of KS is not fully understood, evidence points to its induction by a specific infectious agent. A predisposition for the disease is presumed [1]. Clinically, KS is manifested as an acute febrile form of vasculitis, which may be fatal if not diagnosed at an early stage. Other signs of the disease include dilatation of the conjunctival vessels and iridocyclitis. These frequent ocular manifestations may be of diagnostic importance, since in $45 \%$ of instances the typical systemic criteria are not completely fulfilled. Although two-thirds of cases present with bilateral conjunctival injection and/or iridocyclitis, the vast majority of patients are not referred to an ophthalmologist, thereby delaying the final diagnosis [2].

Histologically, granulomatous arteriitis is observed within a week of the onset of KS: layers of the vascular walls are implicated, and the condition has spread to the surrounding tissue. Dilatation of the coronary arteries follows frequently, on which basis the diagnosis is confirmed. However, by this time, severe, irreversible vascular damage has been incurred, and all arteries of the body are presumed to be simultaneously implicated [1].

J. G. Garweg $(\bowtie)$

Clinic for Uveitis, Swiss Eye Institute and University of Bern,

Bern CH-3012, Switzerland

e-mail: justus.garweg@eye-institute.ch

C. Suter $\cdot$ C. Aebi-Ochsner $\cdot$ R. Villiger

Children's Hospital Wildermeth,

Biel, Switzerland
A wide spectrum of ocular manifestations has been reported (see Table 1). While anterior segment involvement represents a typical diagnostic feature in KS, changes within the posterior segment - mainly papilloedema - have been only seldom documented [3, 4]. Since posterior segment involvement is probably not a rare occurrence in KS, the scarcity of reported findings might reflect difficulties in examining the retinae of these impaired and extremely light-sensitive patients rather than an absence of pathological changes per se. Herein, we present retinal findings for a child with KS and macular oedema that were obtained by non-invasive, non-lightdependent optical coherence tomography (OCT), and provide an overview of posterior segment involvement in KS.

\section{Case report}

A previously healthy 6 -year-old boy presented with a 5-day history of fever up to $40^{\circ} \mathrm{C}$, a skin rash and pain in the ankle joints, mainly on the right side. The child had symmetrically red and painful eyes, which were extremely sensitive to light.

On medical examination the boy was in a generally reduced condition and barely able to walk. With a body temperature of $37.7^{\circ} \mathrm{C}$, all vital parameters were normal. A maculopapular skin rash, with some plaques on the extremities and the trunk, was observed. The scrotum was slightly red, but not sore; both ankle joints were swollen and tender. The boy manifested bilateral cervical lymphadenopathy and bilateral conjunctival hyperaemia, as well as dry and red lips with mild inflammation of the throat and a strawberry tongue. Otherwise, his physical state was unremarkable. Blood analysis revealed an elevated erythrocyte sedimentation rate $(104 \mathrm{~mm} / \mathrm{hour})$, an increased level of the C-reactive protein $(64 \mathrm{mg} / \mathrm{l})$, mild anaemia $(\mathrm{Hb}$ $107 \mathrm{~g} / \mathrm{l})$ and marked thrombocytosis $(823 \mathrm{~g} / \mathrm{l})$. However, the white-blood-cell count was within normal limits. Urine 
Table 1 Manifestations found in the literature

\begin{tabular}{|c|c|c|c|}
\hline \multicolumn{2}{|l|}{ References } & \multirow{2}{*}{$\begin{array}{l}\text { Number of cases } \\
2 \text { of } 18 \text { children }\end{array}$} & \multirow{2}{*}{$\begin{array}{l}\text { Manifestation } \\
\text { Bilateral optic disc swelling and bilateral vitreous opacities }\end{array}$} \\
\hline Ohno et al. 1982 [7] & Prospective study & & \\
\hline Jacob et al. 1982 [3] & Prospective study & 1 of 9 children & Unilateral choroidal, retinal and vitreous changes \\
\hline Font et al. 1983 [11] & Case report/ post mortem findings & 1 & Bilateral inner retinal ischemia \\
\hline Anand and Yang 2004 [9] & Case report & 1 & $\begin{array}{l}\text { Bilateral optic disc swelling, perivascular sheathing, leakage } \\
\text { from the disc vessels }\end{array}$ \\
\hline Farvardin et al. 2007 [5] & Case report & 1 & Diffuse retinal whitening, narrowing of arterioles, disc swelling \\
\hline Grouteau et al. 2011 [4] & Case report & 1 & Vitritis, bilateral optic disc swelling with papillitis \\
\hline Own case & Case report & 1 & $\begin{array}{l}\text { Epithelial keratitis, granulomatous anterior uveitis, retinal } \\
\text { vasculitis with diffuse macular edema and stellate maculopathy, } \\
\text { ischaemic retinopathy, optic disc involvement }\end{array}$ \\
\hline
\end{tabular}

analysis, electrocardiography, and echocardiography disclosed no abnormalities. Based on these findings, KS was diagnosed. Intravenous immunoglobulin ( $2 \mathrm{~g} / \mathrm{kg}$ of body weight) was promptly initiated, and high doses of aspirin $(80 \mathrm{mg} / \mathrm{kg}$ of body weight/day) were prescribed.

On the second day of treatment, the child was afebrile, but complained of increasing pain in his eyes. Vision of the right eye was reduced due to corneal opacity. The boy was referred for an ophthalmological evaluation. Dermatitis of the lids with crusty blepharitis, conjunctival hyperaemia, and punctuate epithelial keratitis with endothelial precipitates were identified, more in the right eye than in the left. Fundoscopy revealed the optic discs to be widely normal, but horizontal retinal folds were observed in the papillomacular bundle of both eyes, with diffuse macular oedema, perifoveal stellate exudates, and cotton-wool spots along the vascular arcades, as well as mild venous dilatation without evident retinal vasculitic changes or choroideal involvement (Fig. 1). OCT confirmed the macular oedema, and additionally revealed subfoveal detachment of the pigmented epithelium, as well as hyper-reflective structures,

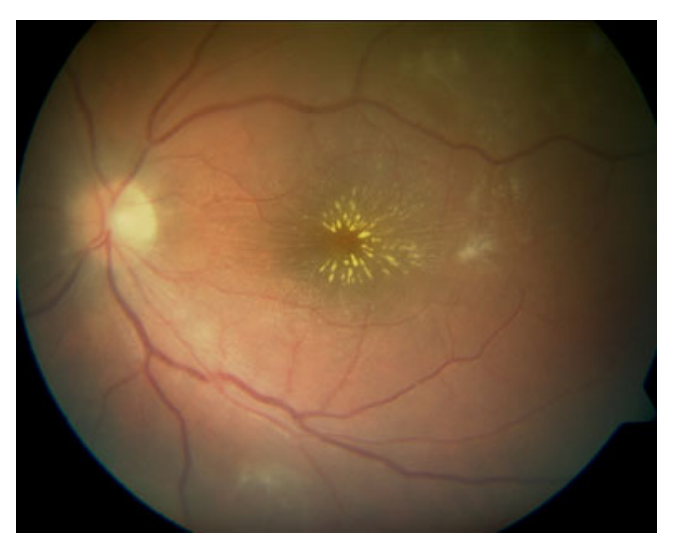

Fig. 1 This image reveals the optic-nerve head to have a normal appearance. The macula, on the other hand, evinces signs of diffuse oedema and contains prominent stellate precipitates. Ischemic changes are observed temporal to the macula and along the arcade of inferotemporal vessels, which are themselves also mildly implicated which were consistent with the presence of lipid exudates in the outer nuclear layer. At the site of the cotton-wool spots, diffuse tissue hyper-reflectivity and an accumulation of intraretinal fluid were identified - findings which were consistent with ischaemic occlusion of the capillaries and severe damage to the uveo-vascular barrier (Fig. 2).
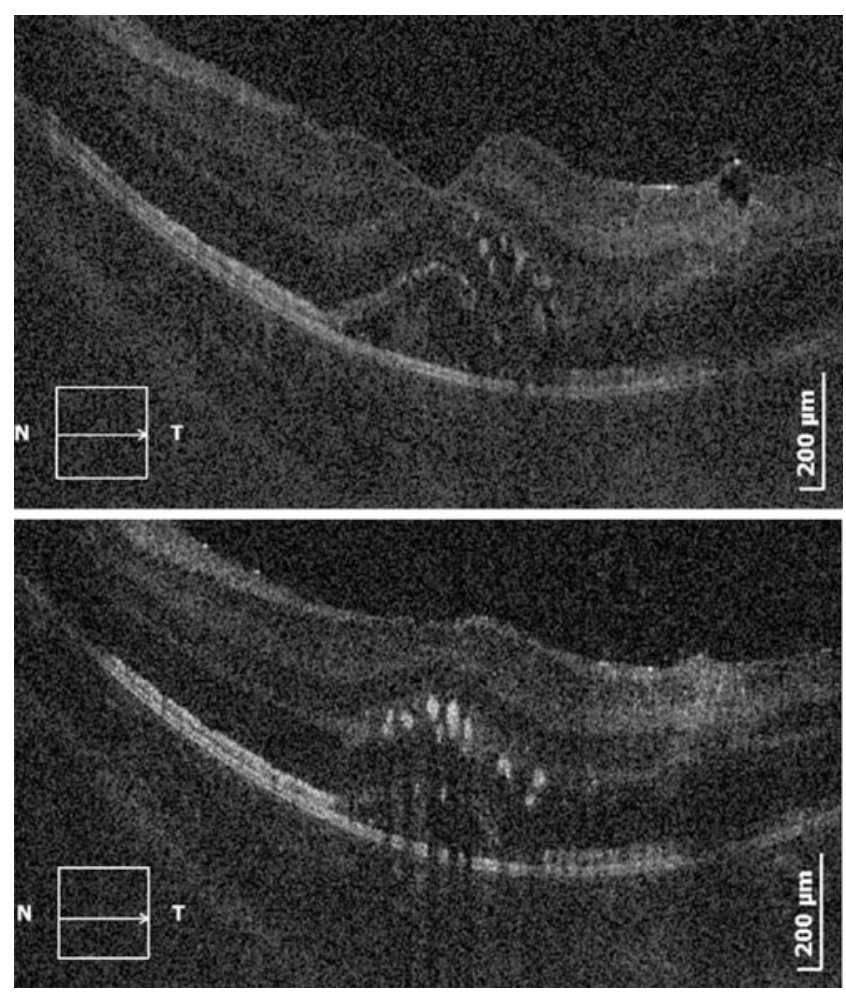

Fig. 2 The upper image represents an OCT section through the foveal region. It reveals subfoveal detachment of the pigmented epithelium and hyper-reflective spots in the inner nuclear layer, as well as diffuse thickening and mild hyper-reflectivity of the inner retinal layers temporally. The lower image, which was taken $200 \mu \mathrm{m}$ below the upper one, reveals an increase in the density of the hyper-reflective spots at the margin of the foveal avascular zone. Within the clinically visible cotton-wool spot, the diffuse hyper-reflectivity and the smudgy delineation of the neuroretinal layers are a consequence of focal damage in the parafoveal region 
Due to the ocular involvement, prednisolone was administered locally and systemically, the initial dose $(1 \mathrm{mg} / \mathrm{kg}$ of body weight) being tapered over 14 days. On day 4, the child was discharged from the hospital. A prophylactic dose of aspirin was continued. The boy's general condition had greatly improved, and he was no longer feverish. The skin rash had almost disappeared, and the ankles had a normal appearance. Although the eyes were no longer painful, mild conjunctivitis was still apparent bilaterally, and mild subepithelial opacity of the cornea persisted in the right globe. Within 2 weeks, the visual acuity of both eyes had returned to $20 / 20$, and the OCT findings were normal. Repeated echocardiography revealed no signs of coronary involvement up to the third month, after which time monitoring was discontinued.

\section{Discussion}

In this case of a 6-year-old child with KS, bilateral macular oedema and stellate maculopathy together with lipid exudation were observed in the absence of optic-disc swelling. Hitherto, ocular manifestations of the disease have been described in detail only by Jacob et al. using classical analytical tools (3). By implementing OCT, we have demonstrated for the first time in vivo vascular leakage and defects in retinal microperfusion. Given that many middlesized arteries throughout the body are simultaneously implicated in the pathogenesis of KS [1], the routine application of OCT could afford a sensitive and noninvasive means of detecting macular oedema and retinovascular involvement in severely affected children, without subjecting them to further light stress. Since the retinal abnormalities may not necessarily respond to high doses of aspirin and intravenous immunoglobulin, which constitute the established course of treatment for KS [4], adjunctive therapy with corticosteroids may be needed to tackle the posteriorsegment affection and to avert permanent visual loss due to vascular occlusion in this region [5].

The most frequently encountered ocular manifestations of bilateral non-exudative conjunctivitis, superficial keratitis and anterior uveitis [6-9], were also observed in our patient (Fig. 1). In contrast to other cases, our patient did not present optic-nerve-head oedema. In a prospective study of 18 children with KS [7], which was conducted in 1982, bilateral swelling of the optic disc occurred in two patients. And in a likewise prospective study which was conducted by Jacob et al. in the same year, only one instance of posteriorsegment involvement was reported: swelling of the temporal disc margin was observed together with unilateral vitritis, retinal and choroidal pathology - including macular oedema and poor vision [3]. In the context of optic-nerve-head swelling, vascular leakage has been detected by fluorescence angiography [9]. However, this light-intensive technique is usually poorly tolerated by young and diseased children, and its application is not primarily justified from the point of view of its therapeutic implications [4]. Although OCT cannot assess changes in the choroidal vasculature, which have been occasionally reported in the context of $\mathrm{KS}$ [3, 10], our observations correlate well with post-mortem findings of bilateral innerretinal ischaemia [11]. If retinal ischaemia occurs during the acute phase of KS, there is a risk of persistent visual damage [5, 11]. Since the long-term prognosis after recovery from the acute phase is good [12], retinal oedema and damage might be averted by supplementing the established therapy for KS with systemically-administered steroids, which were welltolerated in our patient. If the macula is involved, OCT would help to assess the visual prognosis [13]. The systematic assessment of patients with KS has been recommended, since even in incomplete clinical pictures, $24 \%$ ultimately develop coronary artery aneurisms [2].

Although macular oedema is not specific for KS, in combination with conjunctivitis, photophobia, iritis, and fever, the disease ranks high in the list of differential diagnoses. In childhood, inflammation is a leading cause of increased vascular permeability and of uveovascular-barrier breakdown. The retina may not primarily be implicated, as in the case of iritis. Pathological changes may evolve slowly during the course of several weeks or even months [14]. The diffuse tissue hyper-reflectivity that results from the exudation of proteins and lipids as well as the accumulation of intraretinal fluid which are a consequence of severe uveovascular barrier damage and ischaemic occlusion of the capillaries in the absence of a long-standing vascular disease - are specific for KS.

In conclusion, we recommend an ocular evaluation of all patients with KS, as well as a routinely performed OCTassessment of the macula to reveal its involvement and the existence of retinal ischaemia in cases with posterior segment involvement.

Financial disclosure None of the authors has either a financial or a proprietary interest in any of the presented materials or methods.

\section{References}

1. Takahashi K, Oharaseki T, Yokouchi Y (2011) Pathogenesis of Kawasaki disease. Clin Exp Immunol 164(Suppl 1):20-22

2. Blatt AN, Vogler L, Tychsen L (1996) Incomplete presentations in a series of 37 children with Kawasaki disease: the role of the pediatric ophthalmologist. J Pediatr Ophthalmol Strabismus 33:114-119

3. Jacob JL, Polomeno RC, Chad Z, Lapointe N (1982) Ocular manifestations of Kawasaki disease (mucocutaneous lymph node syndrome). Can J Ophthalmol 17:199-202

4. Grouteau E, Debuisson C, Brochard K, Paranon S, Lesage Beaudon C, Pajot C, Claudet I (2011) Severe global inflammatory involvement 
of ocular segments and optic disc swelling in a 12-year-old girl with Kawasaki disease. Eur J Ophthalmol 21:112-114

5. Farvardin M, Kashef S, Aleyasin S, Nabavizadeh SH, Sajjadi M, Safari M (2007) Sudden unilateral blindness in a girl with Kawasaki disease. J Pediatr Ophthalmol Strabismus 44:303304

6. Burke MJ, Rennebohm RM (1981) Eye involvement in Kawasaki disease. J Pediatr Ophthalmol Strabismus 18:7-11

7. Ohno S, Miyajima T, Higuchi M, Yoshida A, Matsuda H, Saheki Y, Nagamatsu I, Togashi T, Matsumoto S (1982) Ocular manifestations of Kawasaki's disease (mucocutaneous lymph node syndrome). Am J Ophthalmol 93:713-717

8. Smith LB, Newburger JW, Burns JC (1989) Kawasaki syndrome and the eye. Pediatr Infect Dis J 8:116-118

9. Anand S, Yang YC (2004) Optic disc changes in Kawasaki disease. J Pediatr Ophthalmol Strabismus 41:177-179
10. Googe JM Jr, Brady SE, Argyle JC, Apple DJ, Gooch WM 3rd (1985) Choroiditis in infantile periarteritis nodosa. Arch Ophthalmol 103:81-83

11. Font RL, Mehta RS, Streusand SB, O'Boyle TE, Kretzer FL (1983) Bilateral retinal ischemia in Kawasaki disease. Postmortem findings and electron microscopic observations. Ophthalmology 90:569-577

12. Burke MJ, Rennebohm RM, Crowe W, Levinson JE (1981) Followup ophthalmologic examinations in children with Kawasaki's disease. Am J Ophthalmol 91:537-539

13. Roesel M, Henschel A, Heinz C, Dietzel M, Spital G, Heiligenhaus A (2009) Fundus autofluorescence and spectral domain optical coherence tomography in uveitic macular edema. Graefes Arch Clin Exp Ophthalmol 247:1685-1689

14. Scholl S, Augustin A, Loewenstein A, Rizzo S, Kupperman B (2010) General pathophysiology of macular edema. Eur J Ophthalmol S6:10-19 